International Journal of Current Advanced Research

ISSN: O: 2319-6475, ISSN: P: 2319 - 6505, Impact Factor: SJIF: 5.995

Available Online at www.journalijcar.org

Volume 6; Issue 5; May 2017; Page No. 3574-3577

DOI: http://dx.doi.org/10.24327/ijcar.2017.3577.0318

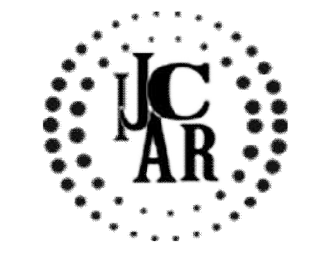

Review Article

\title{
FACTORS INFLUENCING THE DENTINBOND STRENGTH -A REVIEW
}

\section{Aparna M and Sandhya}

Saveetha Dental College, Chennai

\begin{tabular}{l}
\hline A R T I C L E I N F O \\
\hline Article History: \\
Received $8^{\text {th }}$ February, 2017 \\
Received in revised form $12^{\text {th }}$ March, 2017 \\
Accepted $2^{\text {nd }}$ April, 2017 \\
Published online $28^{\text {th }}$ May, 2017
\end{tabular}

Key words:

Dentinbond Strength

\begin{abstract}
A B S T R A C T
Aim: To review on the factors influencingthe dentin bond strength.

Background: The ability of dental professionals to bond resin materials to enamel and dentin has changed the concepts of cavity preparation, caries prevention, and cementation of fixed prostheses. Various modifications are attempted to improve the bond strength of the dentin bonding agent. Some of them include deities of chlorhexidine, chiromancy, etc. This article reviews the factors influencingthe bond strength of dentin.
\end{abstract}

Reason: To create awareness about the various the factors influencingthe bond strength of dentin.

Copyright $\bigcirc 2017$ Aparna M and Sandhya. This is an open access article distributed under the Creative Commons Attribution License, which permits unrestricted use, distribution, and reproduction in any medium, provided the original work is properly cited.

\section{INTRODUCTION}

Today every focus is diverted to conserve tooth structure using restorative materials, which adhere to tooth structure by minimal intervention and are tooth coloured to provide aesthetics. Strong adhesion between the tooth and restorative material would not only eliminate the need for salvaging sound tooth structure for retention form, but also prevent the formation of marginal gaps occurring due to polymerisation stress.

Adhesion of restorative material to enamel has become a routine and reliable aspect of modern restorative dentistry, but adhesion to the dentin has proved to be more difficult and less predictable. ${ }^{[4]}$ Dentin being a living tissue, poses greater obstacles to adhesive bonding than does enamel. Much of the difficulty in bonding to dentin is due to its tubular and heterogenous nature, high fluid content, presence of smear layer, and the need to achieve the pupal biocompatibility. ${ }^{[4],[5],[6]}$ In the beginning, separate chemical components and several application steps were needed for priming and bonding. ${ }^{[7]}$ Current advances have focused on the development of delivery systems that simplify the steps involved. ${ }^{[8]}$

The most recent innovation in dental adhesive technology involves the introduction of "all-in-one" or "self-etch" adhesive systems. The self-etch adhesives no longer need an "etch and rinse phase," but are capable of conditioning the tooth surface and simultaneously preparing it for adhesion. This not only lessens clinical application time, but also significantly reduces the technique-sensitivity or the risk of

*Corresponding author: Aparna M

Saveetha Dental College, Chennai making errors during application or manipulation. ${ }^{[2],[4],[6]}$ Even though these systems use fewer separate components, their application still requires that the steps be performed with great attention. A wide range of dentin shear bond strength (SBS) has been reported for these materials that may indicate technical concerns, which are not well identified. Use of these materials remains extremely technique-sensitive. ${ }^{[8],[9]}$ It has been shown that subtle differences in application techniques can affect bond strength. ${ }^{[10],[11]}$ There are various variables affecting bond strength, which have been investigated, such as bonding to moist vs dry dentin, temperature, and humidity, the use of disinfectants and other possible contaminants, the intensity of curing lights, and the depth of dentin.(20-21)

The amount of force required to break the connection between a bonded (dental) restoration and the tooth surface with the failure occurring in or near the adhesive/adherens interface is defined as bond strength. (1) A major goal of modern dental materials research is the development of dental restorative materials that form strong bonds to tooth structures. The acceptance and widespread use of direct-filling composite resin restorative materials over the past 30 years has stimulated research into bonding to tooth structures. (4) The fundamental mechanism of bonding to enamel and dentin is based on an exchange process in which minerals removed from the dental hard tissues are replaced by resin monomers that, upon polymerization, become micro mechanically interlocked in the created porosities.(5)

Bond strengths can predict the longevity of a restoration to some extent. Adhesive systems are selected based on their bond strengths achieved while testing in laboratories. (1) The achievement of high-strength, durable bonds between tooth structure and restorative materials has been a long-term goal of the dental profession. 
Dental adhesive systems are used to promote adhesion between composite resins and dental structure and should present a similar performance on both enamel and dentin.Resin bonding to enamel is considered a durable and predictable clinical procedure. (6)

The micro-mechanical nature of the interaction of dental adhesives with enamel is a result of the infiltration of resin monomers into the microporosities left by acid dissolution of enamel and the subsequent enveloping of the exposed hydroxyapatite crystals with polymerised monomers within pores in the enamel surface. Successful attempts to bond to dentin in a similar fashion have been extensively studied. (5) The durability of the adhesive-dentin interface is related to its quality, based upon the ability of adhesive monomers to occupy the spaces created by the removal of apatite mineral by acid etching and on their ability to envelop the exposed collagen fibrils. After the dentin surface is conditioned, it is recommended that the surface should be maintained in a moist state prior to bonding, which is commonly referred to as wet bonding. The most currently used adhesive system classification is based on the number of steps necessary for clinical application and on interaction with dental hard tissues. Total-etch adhesive systems, which remove the smear layer with phosphoric acid and combine the functions of primer and adhesive in one bottle, have been widely used. 1 Although long-term clinical success has been achieved with total-etch systems,2-3 the demand for simplified application has increased, resulting in the development of self-etching adhesive systems. (9)

While several studies showed that total-etch adhesives yielded enamel bond strength values superior to self-etching adhesives, 10-12 others have reported that there was no significant difference in enamel bond strengths between these two types of adhesive systems.13-18

The reliability of dentin adhesives is dependent upon the quality of the dentin. Variation in dentin depth can have a significant influence on the bond strength of adhesive systems, as the density and number of tubules play important roles in adhesion.(17) In a study investigating the bond strength of self-etching adhesives to enamel, superficial and deep dentin, no statistically significant differences between adhesive bond strength values were observed for superficial and deep dentin.(2) On the other hand, in another study, selfetching and one-bottle adhesives exhibited significantly lower bond strengths when bonded to deep dentin.(17)

A number of studies have investigated the bonding ability of adhesive systems to either enamel, dentin or both. Although such results are of great value for comparative purposes, in clinical situations, the depth of dentin differs in cavity preparation. Most clinically prepared cavities are complex in design and include not only areas of exposed enamel and superficial dentin, but also deep dentinal areas. Since many different adhesive systems are on the market today, it is desirable to use adhesive systems that produce high, uniform bond strengths to all of these dental hard tissues. (5)

The degree of wetness that is ideal for resin dentin bonding varies widely among the different adhesive systems and depends on the incorporated solvents. The bond strength of total-etch adhesives to moist dentin was found to be higher than to dry dentin in previous studies.(4)

\section{Clinical factors which affects dentin bonding}

A number of clinical factors affect the longevity of dentin bonding. Enhancement of bond strength without compromising the biologic and structural integrity of the tooth can provide a solution to the problem. Achieving predictable bonding to dentin has long been a goal and challenge in restorative dentistry. Dentin bonding agents contain hydrophilic monomers as primers along with a solvent such as acetone or ethanol and an adhesive resin. However, the totaletch technique is highly technique sensitive. When etched dentin is dried using air syringe, the collagen fibers collapse and result in molecular arrangement changes. ${ }^{[1]}$ This result is in agreement with the results of many other studies ${ }^{[2],[9],[10]}$ which showed a significant reduction in bond strength.

Moist dentin produced higher bond strength than dry dentin $^{[3],[11],[12]}$ (group IIa and group IIb). The benefit of the wet bonding technique is derived from the ability of water to maintain the collagen framework and intertubular porosity patent for monomer infiltration. ${ }^{[13],[14]}$

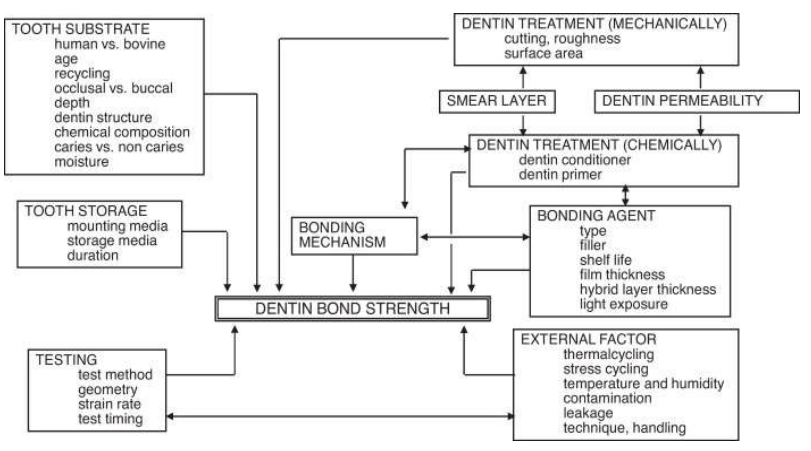

A list of guidelines to help ensure clinical success and longevity of the dentin bond strength are as follows:

\section{Use proper isolation}

Hydrophilic bonding systems may tolerate saliva contamination to a certain degree. 49 However, evidence for such tolerance remains minimal and the mechanism is not well understood, so proper isolation us-ing rubber dams or alternative methods is considered essential to clinical success with cur-rent adhesive systems.(8)

\section{Effect of chlorhexidine}

Chlorohexidine (CHX) is a cationic bisbiguanide, widely known as an antimicrobial agent in oral health. Moreover, it has been reported that CHX is able to inhibit the MMPs' collagenolytic activity, improving the longevity of the bond between adhesives and dentin.[11,12] In fact, Gendron et al.[13] found that the minimum concentrations that are adequate for this inhibition are $0.001 \%$ for MMP-2, $0.02 \%$ for MMP-8, and $0.002 \%$ for MMP-9.There are many factors that influence the bond strength of a restorative material to the dentinal substrate. Mechanical stresses by chewing forces, changes of temperature and $\mathrm{pH}$, water sorption, resin shrinkage, and enzymatic action affect bond integrity to different extents. Moreover, the type and composition of the composite resin and the adhesive system as well as the dentinal substrate are of great importance.[17,18] 


\section{Effect of fluoride}

Fluoride is an important adjuvant in the prevention and treatment of initial caries lesions and its use is indicated in different phases of dental treatment. The formation of white lesions of caries around orthodontic brackets is identified as a common complication found in patients with poor oral hygiene (1). Fluoride gel and sodium fluoride varnish at different concentrations can be used with efficacy and safety for the remineralization of dental structure and to prevent the development of initial caries lesions (2-4). In clinical orthodontics, it is essential to establish a reliable bond strength between enamel and orthodontic appliances. Thus, in vitro studies demonstrate that the use of prophylactic agents with different fluoride concentrations do not significantly affect the adhesion of orthodontic brackets $(9,10)$.

\section{Effect of sodium hypochlorite}

Sodium hypochlorite $(\mathrm{NaOCl})$ and ethylenediaminetetraacetic acid (EDTA) are substances usually used during the endodontic treatment. $\mathrm{NaOCl}$ is an auxiliary irrigant used during root canal instrumentation to promote debridement, lubrication, disinfection, tissue dissolution, collagen layer removal and dentin dehydration ${ }^{7,8}$.

Endodontically treated teeth with a sufficient amount of sound coronary structure should preferably be restored with composite resin by the direct technique, due to its capacity to bond to dentin and increase the fracture resistance of the remaining dental structure ${ }^{11}$. This process requires appropriate interaction of the adhesive system with the dentin substrate However, the irrigating substances frequently used during the endodontic treatment could interfere in the bond strength of the composite resin to dentin.

\section{Surface area}

Whenever a restoration is bonded to dentin, the adjacent enamel should've etched. Years of experience have proved that enamel etching is a very reliable method of bond-ing resins to tooth structure. In addition, when bevels are used, they provide a gradual transition of composite material onto the tooth and thus abetter esthetic result.(5)

\section{Roughen sclerotic dentin}

Bonded restorations of more likely to fail when they are bonded to highly sclerotic dentin) ${ }^{\circ}$ Light roughening with a diamond or carbide bur may provide more micro mechanical locking between resin and den-tin. While not encountered as frequently in paediatric dentistry as in adult restorative dentistry, sclerotic dentin is encountered beneath some carious lesions.(4)

\section{Use mechanical retention.}

With adhesive restorative materials, supplemental mechanical retention (pins, grooves, slots) is frequently to necessary. However, the operator should use mechanical retention in cases where adhesive bonding may not be sufficient to retain or properly seal a restoration.(6)

\section{Wetness of dentin}

Virtually all present-day dentin adhesives bond to dentin that is at least slightly moist. Systems that contain acetone primers are particularly well suited foreboding to wet surfaces, 36' 45 although the optimum degree of surface moistness varies with specific products. However, as a general rule, den-tin should not be desiccated. If dentin is dried-excessively to check the enamel etch, it should be remoistened to improve bond strengths.(7)

\section{Influence of solvents}

Dentin primers and fifth-generation primer/adhesives must be applied in adequate quantity. Some materials re-quire multiple coats, and others probably benefit from application of multiple coats or longer application times. So, solvents must be driven off completely with compressed air before the bonding agent or composite is applied.(11)

\section{Thickness of bonding agent}

Application of the resin bonding agent is the simplest step-in a three-step bonding sequence. However, if the resin is aggressively air-thinned, oxygen inhibition prevents complete polymerisationand-results in lower bond strengths. Thinning the bonding agent with a dry brush is better than thinning with compressed air blasts. For direct composite restorations, the bonding agent should be light-cured before the restorative material is placed to optimise thebonding system's performance.(16)

\section{Use a flexible restorative system}

Flexible restorative materials (e.g., microfilm composites) and"stress-breaking liners" (filled bonding resins) may improve the marginal quality of bonded restorations by compensating for stresses generated by polymerisation shrinkage and tooth flexure. (15)

\section{Fill incrementally}

M1 composites shrink during polymerisation, One method of reducing overall polymerisation shrinkage is to place and cure composite in increments. Although there is now-some controversy about whether this technique provides better marginal adaptation, 57' 58 it still seems advisable, and is necessary when composite thickness exceeds $2 \mathrm{~mm}$ (to provide for adequate light curing).(12)

\section{CONCLUSION}

Advances in adhesive dental technology have radically changed restorative dentistry. Adhesive dentistry is a rapidly evolving discipline. For many years, the dental profession has strived to achieve good adhesion for resin composite to tooth substrate. ${ }^{[3]}$ Therefore, one of the primary objectives of researchers is to achieve a strong, durable, predictable bond, which not only is essential from mechanical stand point but also from biological and esthetic perspectives. For this purpose, SBS was tested at the bonding interface between parent material and the deciduous dentin surface because most masticatory forces are of a shearing nature. ${ }^{[14]}$

Generations of dentin-bonding agents (DBAs) have been developed on the basis of complete understanding of the nature of tooth substance, yet a perfect bonding system and application technique is still elusive. ${ }^{[1]}$ Therefore this review concluded that subtle differences in application techniques can affect bond strength. ${ }^{[10],[11]}$ The acid-etch technique for enamel bonding led to the development of revolutionary restorative, preventive, and aesthetic treatment methods. More recently, developments due some interesting factors like mouth washes which helps in increasing the bond strength, 
dentin bonding have moved adhesive dentistry uneven higher level. Many systems are now available tolerably and durably bond resin to dentin. However, these systems must be used properly to optimisetheir clinical performance.(5)

\section{References}

1. P Pranau Vanajasan, Malarvizhi Dhakshinamoorthy, and CV Subba Rao, Factors affecting the bond strength of self-etch adhesives: A meta-analysis of literature journal of conservative dentistry, Jan-mar 2014 vol 14 issue 1,324-565.

2. Amit Bharadwaj, Comparative evaluation of shear bond strength and rebonding characteristics of halogen curing light unit, journal of orthodontics, October- Dec 2013, issue 2,476-489

3. Iffat Nasim, Prasanna Neelakantan \& C. V. Subbarao, Effect of gutta-percha solvents on the bond strength of two resin-based sealers to root canal dentin journal of Taylor and Francis online, vol 72 issue 14.29-34

4. Prasanna Neelakantan, impact of root dentine conditioning on sealing ability and push-out bond strength of an epoxy resin root canal sealer, International Endodontic journal vol 44, issue 2011, pages 491-498.

5. Shanmugaraj, -Rinsing Procedure on Adhesion of Composite to dentin, journal of pearl dent, vol 4, issue 2,2013.356-465.

6. DhanalakShmi RavikumaR1, Shanmugavel kaRthikeyan2, emg SubRamanian3, Deepa guRunathanDOI:Effect of Mechanical and Chemical Root Surface Treatment on the Shear Bond Strength of Intracanal Post in Primary Anterior Teeth: An In vitro Study.10.7860/JCDR/2017/22117.9182

7. Neelakantan, Prasanna; Sanjeev, Kavitha; Rao, C. V. Subba Source: Ultra morphological Characterization of the Resin Dentin Interface - An in vitro Analysis of Nanoleakage Patterns of Dentin Adhesives.: Journal of Clinical Pediatric Dentistry. 2009, Vol. 33 Issue 3, p223-230.

8. N Ravikumar, P Shankar, and R IndiraShear bond strengths of two dentin bonding agents with two desensitisers:an in vitro study, journal of conservative dentistry 2011,volume 14 ,issue, 247-251

9. E.D Bonilla,Technique-sensitivity of dentin-bonding agent application: The effect on shear bond strength using one-step self-etch adhesive in primary molars: An in vitro study, Journal of Indian Society of Pedodontics and Preventive Dentistry, Vol. 28, No. 3, July-September, 2010, pp. 183-188 T
10. Sarr M, Kane AW, Vreven J, Mine A, Van Landuyt KL, Peumans M, et al. Microtensile bond strength and interfacial characterization of 11 contemporary adhesives bonded to bur-cut dentin. Oper Dent. 2010; 35:94-104.

11. Salz U, Bock T. Adhesion performance of new hydrolytically stable one-component self-etching enamel/dentin adhesives. $J$ Adhes Dent. 2010; 12:7-10.

12. Gianini RJ, do Amaral FL, Flório FM, Basting RT. Microtensile bond strength of etch-and-rinse and selfetch adhesive systems to demineralized dentin after the use of a papain-based chemomechanical method. Am J Dent. 2010;23:23-8.

13. Kimmes NS, Barkmeier WW, Erickson RL, Latta MA. Adhesive bond strengths to enamel and dentin using recommended and extended treatment times. Oper Dent. 2010;35:112-9

14. Pashley DH, Tay FR, Breschi L, Tjaderhane L, Carvalho RM, Carrilho M, Tezvergil-Mutluay A: State of the art etch-and-rinse adhesives. Dent Mater 2011, 27: 1-16.

15. Van Meerbeek B, Yoshihara K, Yoshida Y, Mine A, De Munck J, Van Landuyt KL: State of the art of selfetch adhesives. Dent Mater 2011, 27: 17-28.

16. Münchow EA, Bossardi M, Priebe TC, Valente LL, Zanchi $\mathrm{CH}$, Ogliari FA, Piva E: Microtensile versus microshear bond strength between dental adhesives and the dentin substrate. Int $J$ Adhes Adhes 2013, 46: 9599.

17. Costa TR, Ferreira SQ, Klein-Junior CA, Loguercio $\mathrm{AD}$, Reis A: Durability of surface treatments and intermediate agents used for repair of a polished composite. Oper Dent 2010, 35: 231-237.

18. Peumans M, De Munck J, Van Landuyt KL, Poitevin A, Lambrechts P, Van Meerbeek B: Eight-year clinical evaluation of a 2-step self-etch adhesive with and without selective enamel etching. Dent Mater 2010, 26: 1176-1184.

19. Ricardo M. Carvalho1,*, Adriana P. Manso1, Saulo Geraldeli2, Franklin R. Tay3, and David H. PashleyDurability of bonds and clinical success of adhesive restorations NIH PUBLIC ACCESS, dent mat $2012,28-35$.

20. Perdigao J. Dentin bonding-variables related to the clinical situation and the substrate treatment. Dent Mater. 2010; 26:e24-37.

\section{How to cite this article:}

Aparna M and Sandhya (2017) ' Factors Influencing The Dentinbond Strength -A Review', International Journal of Current Advanced Research, 06(05), pp. 3574-3577.

DOI: http://dx.doi.org/10.24327/ijcar.2017.3577.0318 